Internet Engineering Task Force (IETF)

Request for Comments: 6840

Updates: 4033, 4034, 4035, 5155

Category: Standards Track

ISSN: $2070-1721$
S. Weiler, Ed. SPARTA, Inc. D. Blacka, Ed. Verisign, Inc. February 2013

Clarifications and Implementation Notes for DNS Security (DNSSEC)

Abstract

This document is a collection of technical clarifications to the DNS Security (DNSSEC) document set. It is meant to serve as a resource to implementors as well as a collection of DNSSEC errata that existed at the time of writing.

This document updates the core DNSSEC documents (RFC 4033, RFC 4034, and RFC 4035) as well as the NSEC3 specification (RFC 5155). It also defines NSEC3 and SHA-2 (RFC 4509 and RFC 5702) as core parts of the DNSSEC specification.

Status of This Memo

This is an Internet Standards Track document.

This document is a product of the Internet Engineering Task Force (IETF). It represents the consensus of the IETF community. It has received public review and has been approved for publication by the Internet Engineering Steering Group (IESG). Further information on Internet Standards is available in Section 2 of RFC 5741.

Information about the current status of this document, any errata, and how to provide feedback on it may be obtained at http://www.rfc-editor.org/info/rfc6840. 
Copyright Notice

Copyright (c) 2013 IETF Trust and the persons identified as the document authors. All rights reserved.

This document is subject to BCP 78 and the IETF Trust's Legal Provisions Relating to IETF Documents

(http://trustee.ietf.org/license-info) in effect on the date of publication of this document. Please review these documents carefully, as they describe your rights and restrictions with respect to this document. Code Components extracted from this document must include Simplified BSD License text as described in section $4 . e$ of the Trust Legal Provisions and are provided without warranty as described in the Simplified BSD License.

This document may contain material from IETF Documents or IETF Contributions published or made publicly available before November 10, 2008. The person(s) controlling the copyright in some of this material may not have granted the IETF Trust the right to allow modifications of such material outside the IETF Standards Process. Without obtaining an adequate license from the person(s) controlling the copyright in such materials, this document may not be modified outside the IETF standards Process, and derivative works of it may not be created outside the IETF Standards Process, except to format it for publication as an RFC or to translate it into languages other than English. 
Table of Contents

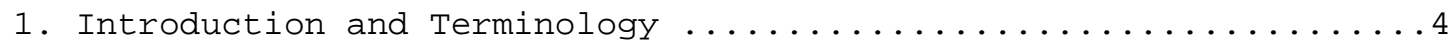

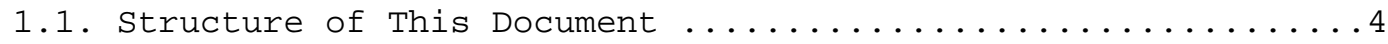

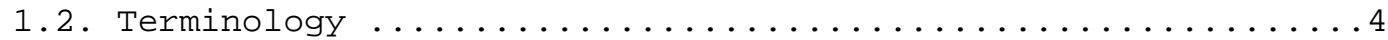

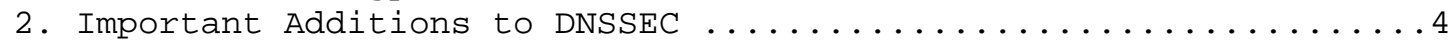

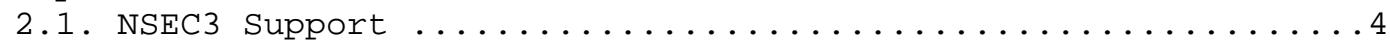

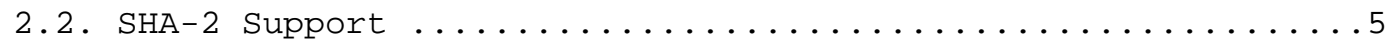

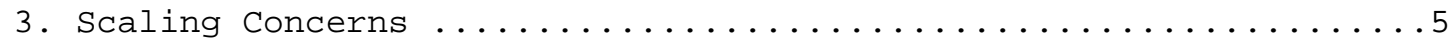

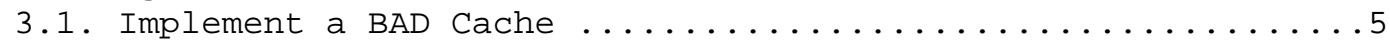

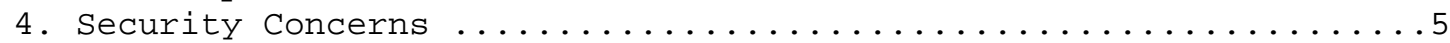

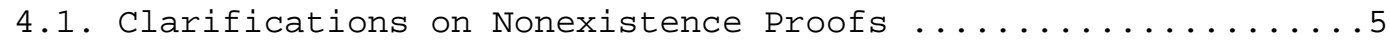

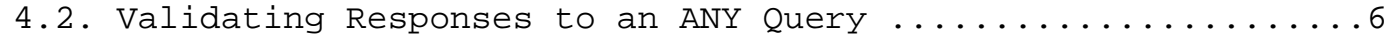

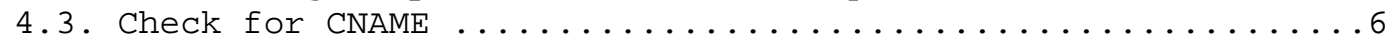

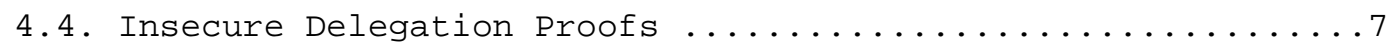

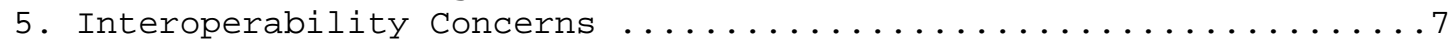

5.1. Errors in Canonical Form Type Code List ..............

5.2. Unknown DS Message Digest Algorithms ...............

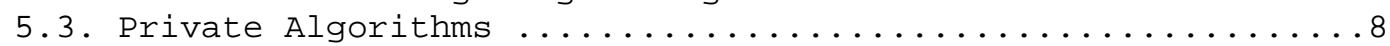

5.4. Caution about Local Policy and Multiple RRSIGs .........9

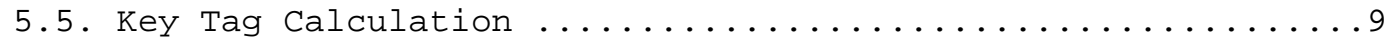

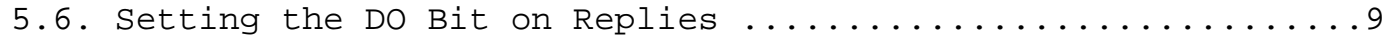

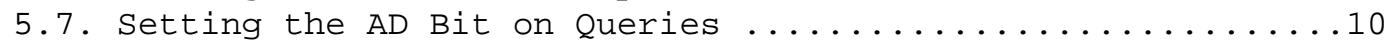

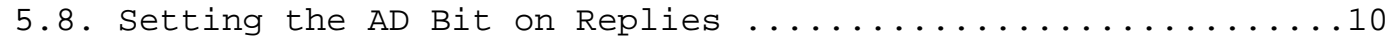

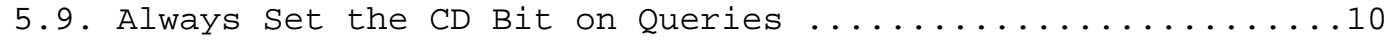

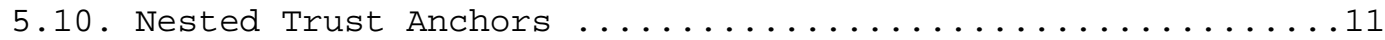

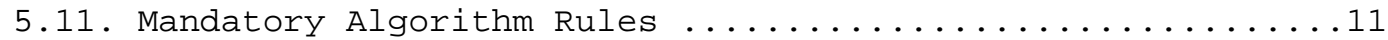

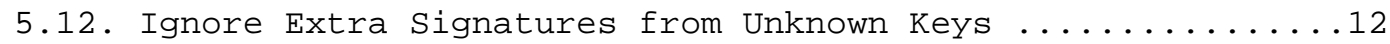

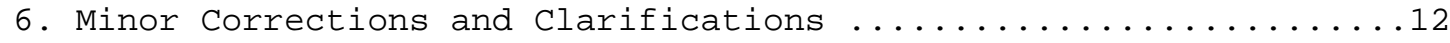

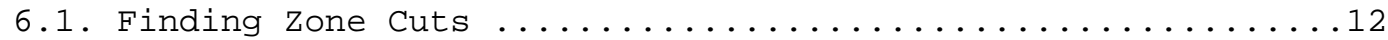

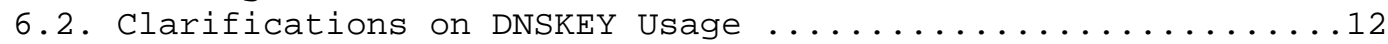

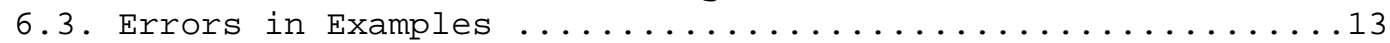

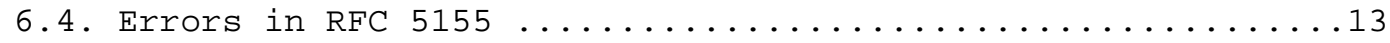

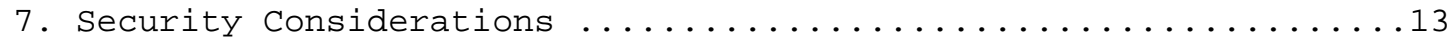

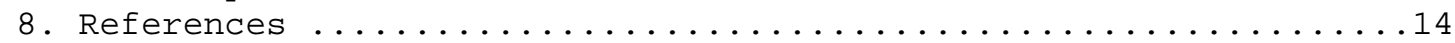

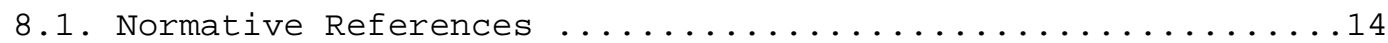

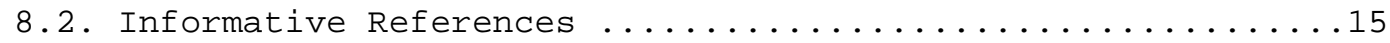

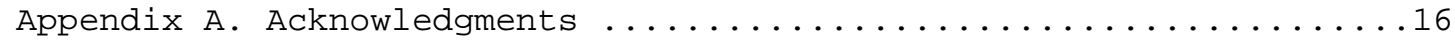

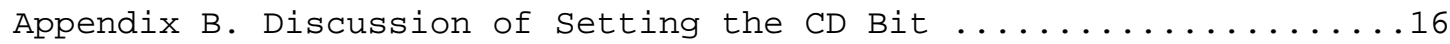

Appendix C. Discussion of Trust Anchor Preference Options .......19

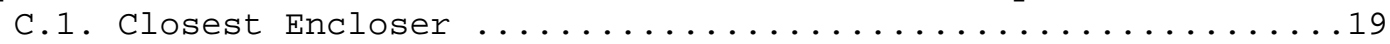

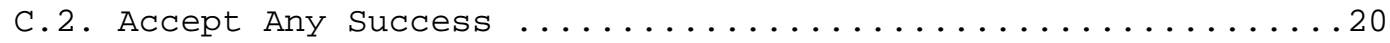

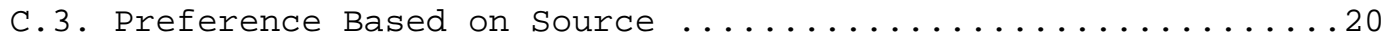


1. Introduction and Terminology

This document lists some additions, clarifications, and corrections to the core DNSSEC specification, as originally described in [RFC4033], [RFC4034], and [RFC4035], and later amended by [RFC5155]. (See Section 2 for more recent additions to that core document set.)

It is intended to serve as a resource for implementors and as a repository of items existing at the time of writing that need to be addressed when advancing the DNSSEC documents along the Standards Track.

\subsection{Structure of This Document}

The clarifications and changes to DNSSEC are sorted according to their importance, starting with ones which could, if ignored, lead to security problems and progressing down to clarifications that are expected to have little operational impact.

\subsection{Terminology}

The key words "MUST", "MUST NOT", "REQUIRED", "SHALL", "SHALL NOT", "SHOULD", "SHOULD NOT", "RECOMMENDED", "NOT RECOMMENDED", "MAY", and "OPTIONAL" in this document are to be interpreted as described in [RFC2119].

2. Important Additions to DNSSEC

This section lists some documents that are now considered core DNSSEC protocol documents in addition to those originally specified in Section 10 of [RFC4033].

\subsection{NSEC3 Support}

[RFC5155] describes the use and behavior of the NSEC3 and NSEC3PARAM records for hashed denial of existence. Validator implementations are strongly encouraged to include support for NSEC3 because a number of highly visible zones use it. Validators that do not support validation of responses using NSEC3 will be hampered in validating large portions of the DNS space.

[RFC5155] is now considered part of the DNS Security Document Family as described by Section 10 of [RFC4033]. 
Note that the algorithm identifiers defined in [RFC5155] (DSA-NSEC3SHA1 and RSASHA1-NSEC3-SHA1) and [RFC5702] (RSASHA256 and RSASHA512) signal that a zone might be using NSEC3, rather than NSEC. The zone may be using either, and validators supporting these algorithms MUST support both NSEC3 and NSEC responses.

\subsection{SHA-2 Support}

[RFC4509] describes the use of SHA-256 as a digest algorithm in Delegation Signer (DS) RRs. [RFC5702] describes the use of the RSASHA256 and RSASHA512 algorithms in DNSKEY and RRSIG RRs.

Validator implementations are strongly encouraged to include support for these algorithms for DS, DNSKEY, and RRSIG records.

Both [RFC4509] and [RFC5702] are now considered part of the DNS Security Document Family as described by section 10 of [RFC4033].

\section{Scaling Concerns}

\subsection{Implement a BAD Cache}

Section 4.7 of [RFC4035] permits security-aware resolvers to implement a BAD cache. That guidance has changed: security-aware resolvers SHOULD implement a BAD cache as described in [RFC4035].

This change in guidance is based on operational experience with DNSSEC administrative errors leading to significant increases in DNS traffic, with an accompanying realization that such events are more likely and more damaging than originally supposed. An example of one such event is documented in "Rolling Over DNSSEC Keys" [Huston].

4. Security Concerns

This section provides clarifications that, if overlooked, could lead to security issues.

\subsection{Clarifications on Nonexistence Proofs}

Section 5.4 of [RFC4035] under-specifies the algorithm for checking nonexistence proofs. In particular, the algorithm as presented would allow a validator to interpret an NSEC or NSEC3 RR from an ancestor zone as proving the nonexistence of an $R R$ in a child zone.

An "ancestor delegation" NSEC RR (or NSEC3 RR) is one with:

o the NS bit set,

o the start of Authority (SOA) bit clear, and 
- a signer field that is shorter than the owner name of the NSEC RR, or the original owner name for the NSEC3 RR.

Ancestor delegation NSEC or NSEC3 RRS MUST NOT be used to assume nonexistence of any RRs below that zone cut, which include all RRs at that (original) owner name other than DS RRs, and all RRs below that owner name regardless of type.

Similarly, the algorithm would also allow an NSEC RR at the same owner name as a DNAME RR, or an NSEC3 RR at the same original owner name as a DNAME, to prove the nonexistence of names beneath that DNAME. An NSEC or NSEC3 RR with the DNAME bit set MUST NOT be used to assume the nonexistence of any subdomain of that NSEC/NSEC3 RR's (original) owner name.

\subsection{Validating Responses to an ANY Query}

[RFC4035] does not address how to validate responses when QTYPE=*. As described in section 6.2.2 of [RFC1034], a proper response to QTYPE=* may include a subset of the RRsets at a given name. That is, it is not necessary to include all RRsets at the QNAME in the response.

When validating a response to QTYPE=*, all received RRsets that match QNAME and QCLASS MUST be validated. If any of those RRsets fail validation, the answer is considered Bogus. If there are no RRsets matching QNAME and QCLASS, that fact MUST be validated according to the rules in section 5.4 of [RFC4035] (as clarified in this document). To be clear, a validator must not expect to receive all records at the QNAME in response to QTYPE=*.

\subsection{Check for CNAME}

Section 5 of [RFC4035] says nothing explicit about validating responses based on (or that should be based on) CNAMEs. When validating a NOERROR/NODATA response, validators MUST check the CNAME bit in the matching NSEC or NSEC3 RR's type bitmap in addition to the bit for the query type.

Without this check, an attacker could successfully transform a positive CNAME response into a NOERROR/NODATA response by (for example) simply stripping the CNAME RRset from the response. A naive validator would then note that the QTYPE was not present in the matching NSEC/NSEC3 RR, but fail to notice that the CNAME bit was set; thus, the response should have been a positive CNAME response. 


\subsection{Insecure Delegation Proofs}

Section 5.2 of [RFC4035] specifies that a validator, when proving a delegation is not secure, needs to check for the absence of the DS and SOA bits in the NSEC (or NSEC3) type bitmap. The validator also MUST check for the presence of the NS bit in the matching NSEC (or NSEC3) RR (proving that there is, indeed, a delegation), or alternately make sure that the delegation is covered by an NSEC3 RR with the Opt-Out flag set.

Without this check, an attacker could reuse an NSEC or NSEC3 RR matching a non-delegation name to spoof an unsigned delegation at that name. This would claim that an existing signed RRset (or set of signed RRsets) is below an unsigned delegation, thus not signed and vulnerable to further attack.

5. Interoperability Concerns

5.1. Errors in Canonical Form Type Code List

When canonicalizing DNS names (for both ordering and signing), DNS names in the RDATA section of NSEC resource records are not converted to lowercase. DNS names in the RDATA section of RRSIG resource records are converted to lowercase.

The guidance in the above paragraph differs from what has been published before but is consistent with current common practice. Item 3 of Section 6.2 of [RFC4034] says that names in both of these RR types should be converted to lowercase. The earlier [RFC3755] says that they should not. Current practice follows neither document fully.

Section 6.2 of [RFC4034] also erroneously lists HINFO as a record that needs conversion to lowercase, and twice at that. Since HINFO records contain no domain names, they are not subject to case conversion.

\subsection{Unknown DS Message Digest Algorithms}

Section 5.2 of [RFC4035] includes rules for how to handle delegations to zones that are signed with entirely unsupported public key algorithms, as indicated by the key algorithms shown in those zones' DS RRsets. It does not explicitly address how to handle DS records that use unsupported message digest algorithms. In brief, DS records using unknown or unsupported message digest algorithms MUST be treated the same way as DS records referring to DNSKEY RRs of unknown or unsupported public key algorithms. 
The existing text says:

If the validator does not support any of the algorithms listed in an authenticated DS RRset, then the resolver has no supported authentication path leading from the parent to the child. The resolver should treat this case as it would the case of an authenticated NSEC RRset proving that no DS RRset exists, as described above.

In other words, when determining the security status of a zone, a validator disregards any authenticated DS records that specify unknown or unsupported DNSKEY algorithms. If none are left, the zone is treated as if it were unsigned.

This document modifies the above text to additionally disregard authenticated DS records using unknown or unsupported message digest algorithms.

\subsection{Private Algorithms}

As discussed above, section 5.2 of [RFC4035] requires that validators make decisions about the security status of zones based on the public key algorithms shown in the DS records for those zones. In the case of private algorithms, as described in Appendix A.1.1 of [RFC4034], the eight-bit algorithm field in the DS RR is not conclusive about what algorithm(s) is actually in use.

If no private algorithms appear in the DS RRset, or if any supported algorithm appears in the DS RRset, no special processing is needed. Furthermore, if the validator implementation does not support any private algorithms, or only supports private algorithms using an algorithm number not present in the DS RRset, no special processing is needed.

In the remaining cases, the security status of the zone depends on whether or not the resolver supports any of the private algorithms in use (provided that these DS records use supported message digest algorithms, as discussed in Section 5.2 of this document). In these cases, the resolver MUST retrieve the corresponding DNSKEY for each private algorithm DS record and examine the public key field to determine the algorithm in use. The security-aware resolver MUST ensure that the hash of the DNSKEY RR'S owner name and RDATA matches the digest in the DS RR as described in section 5.2 of [RFC4035], authenticating the DNSKEY. If all of the retrieved and authenticated DNSKEY RRS use unknown or unsupported private algorithms, then the zone is treated as if it were unsigned. 
Note that if none of the private algorithm DS RRs can be securely matched to DNSKEY RRS and no other DS establishes that the zone is secure, the referral should be considered Bogus data as discussed in [RFC4035].

This clarification facilitates the broader use of private algorithms, as suggested by [RFC4955].

\subsection{Caution about Local Policy and Multiple RRSIGs}

When multiple RRSIGs cover a given RRset, Section 5.3.3 of [RFC4035] suggests that "the local resolver security policy determines whether the resolver also has to test these RRSIG RRs and how to resolve conflicts if these RRSIG RRs lead to differing results".

This document specifies that a resolver SHOULD accept any valid RRSIG as sufficient, and only determine that an RRset is Bogus if all RRSIGs fail validation.

If a resolver adopts a more restrictive policy, there's a danger that properly signed data might unnecessarily fail validation due to cache timing issues. Furthermore, certain zone management techniques, like the Double Signature Zone Signing Key Rollover method described in Section 4.2.1.2 of [RFC6781], will not work reliably. Such a resolver is also vulnerable to malicious insertion of gibberish signatures.

\subsection{Key Tag Calculation}

Appendix B.1 of [RFC4034] incorrectly defines the Key Tag field calculation for algorithm 1. It correctly says that the Key Tag is the most significant 16 of the least significant 24 bits of the public key modulus. However, [RFC4034] then goes on to incorrectly say that this is fourth-to-last and third-to-last octets of the public key modulus. It is, in fact, the third-to-last and second-tolast octets.

\subsection{Setting the DO Bit on Replies}

As stated in section 3 of [RFC3225], the DNSSEC OK (DO) bit of the query MUST be copied in the response. However, in order to interoperate with implementations that ignore this rule on sending, resolvers MUST ignore the DO bit in responses. 


\subsection{Setting the AD Bit on Queries}

The semantics of the Authentic Data (AD) bit in the query were previously undefined. Section 4.6 of [RFC4035] instructed resolvers to always clear the $\mathrm{AD}$ bit when composing queries.

This document defines setting the AD bit in a query as a signal indicating that the requester understands and is interested in the value of the $\mathrm{AD}$ bit in the response. This allows a requester to indicate that it understands the $\mathrm{AD}$ bit without also requesting DNSSEC data via the DO bit.

\subsection{Setting the AD Bit on Replies}

Section 3.2.3 of [RFC4035] describes under which conditions a validating resolver should set or clear the $\mathrm{AD}$ bit in a response. In order to interoperate with legacy stub resolvers and middleboxes that neither understand nor ignore the AD bit, validating resolvers SHOULD only set the $A D$ bit when a response both meets the conditions listed in Section 3.2.3 of [RFC4035], and the request contained either a set DO bit or a set $A D$ bit.

\subsection{Always set the CD Bit on Queries}

When processing a request with the Checking Disabled (CD) bit set, a resolver SHOULD attempt to return all response data, even data that has failed DNSSEC validation. Section 3.2.2 of [RFC4035] requires a resolver processing a request with the $\mathrm{CD}$ bit set to set the $\mathrm{CD}$ bit on its upstream queries.

This document further specifies that validating resolvers SHouLD set the CD bit on every upstream query. This is regardless of whether the CD bit was set on the incoming query or whether it has a trust anchor at or above the QNAME.

[RFC4035] is ambiguous about what to do when a cached response was obtained with the CD bit unset, a case that only arises when the resolver chooses not to set the CD bit on all upstream queries, as specified above. In the typical case, no new query is required, nor does the cache need to track the state of the CD bit used to make a given query. The problem arises when the cached response is a server failure (RCODE 2), which may indicate that the requested data failed DNSSEC validation at an upstream validating resolver. ([RFC2308] permits caching of server failures for up to five minutes.) In these cases, a new query with the CD bit set is required.

Appendix B discusses more of the logic behind the recommendation presented in this section. 


\subsection{Nested Trust Anchors}

A DNSSEC validator may be configured such that, for a given response, more than one trust anchor could be used to validate the chain of trust to the response zone. For example, imagine a validator configured with trust anchors for "example." and "zone.example." When the validator is asked to validate a response to

"www.sub.zone.example.", either trust anchor could apply.

When presented with this situation, DNSSEC validators have a choice of which trust anchor(s) to use. Which to use is a matter of implementation choice. Appendix C discusses several possible algorithms.

It is possible and advisable to expose the choice of policy as a configuration option. As a default, it is suggested that validators implement the "Accept Any Success" policy described in Appendix C.2 while exposing other policies as configuration options.

The "Accept Any Success" policy is to try all applicable trust anchors until one gives a validation result of secure, in which case the final validation result is secure. If and only if all applicable trust anchors give a result of Insecure, the final validation result is Insecure. If one or more trust anchors lead to a Bogus result and there is no secure result, then the final validation result is Bogus.

\subsection{Mandatory Algorithm Rules}

The last paragraph of Section 2.2 of [RFC4035] includes rules describing which algorithms must be used to sign a zone. Since these rules have been confusing, they are restated using different language here:

The DS RRset and DNSKEY RRset are used to signal which algorithms are used to sign a zone. The presence of an algorithm in either a zone's DS or DNSKEY RRset signals that that algorithm is used to sign the entire zone.

A signed zone MUST include a DNSKEY for each algorithm present in the zone's DS RRset and expected trust anchors for the zone. The zone MUST also be signed with each algorithm (though not each key) present in the DNSKEY RRset. It is possible to add algorithms at the DNSKEY that aren't in the DS record, but not vice versa. If more than one key of the same algorithm is in the DNSKEY RRset, it is sufficient to sign each RRset with any subset of these DNSKEYs. It is acceptable to sign some RRsets with one subset of keys (or key) and other RRsets with a different subset, so long as at least 
one DNSKEY of each algorithm is used to sign each RRset.

Likewise, if there are DS records for multiple keys of the same algorithm, any subset of those may appear in the DNSKEY RRset.

This requirement applies to servers, not validators. Validators SHOULD accept any single valid path. They SHOULD NOT insist that all algorithms signaled in the DS RRset work, and they MUST NOT insist that all algorithms signaled in the DNSKEY RRset work. A validator MAY have a configuration option to perform a signature completeness test to support troubleshooting.

\subsection{Ignore Extra Signatures from Unknown Keys}

Validating resolvers MUST disregard RRSIGs in a zone that do not (currently) have a corresponding DNSKEY in the zone. Similarly, a validating resolver MUST disregard RRSIGs with algorithm types that don't exist in the DNSKEY RRset.

Good key rollover and algorithm rollover practices, as discussed in RFC 6781 and its successor documents and as suggested by the rules in the previous section, may require that such RRSIGs be present in a zone.

6. Minor Corrections and Clarifications

\subsection{Finding Zone Cuts}

Appendix C.8 of [RFC4035] discusses sending DS queries to the servers for a parent zone but does not state how to find those servers. Specific instructions can be found in section 4.2 of [RFC4035].

\subsection{Clarifications on DNSKEY Usage}

It is possible to use different DNSKEYs to sign different subsets of a zone, constrained only by the rules in section 5.11. It is even possible to use a different DNSKEY for each RRset in a zone, subject only to practical limits on the size of the DNSKEY RRset and the above rules. However, be aware that there is no way to tell resolvers what a particular DNSKEY is supposed to be used for -- any DNSKEY in the zone's signed DNSKEY RRset may be used to authenticate any RRset in the zone. For example, if a weaker or less trusted DNSKEY is being used to authenticate NSEC RRsets or all dynamically updated records, that same DNSKEY can also be used to sign any other RRsets from the zone.

Furthermore, note that the SEP bit setting has no effect on how a DNSKEY may be used -- the validation process is specifically prohibited from using that bit by section 2.1.2 of [RFC4034]. It is 
possible to use a DNSKEY without the SEP bit set as the sole secure entry point to the zone, yet use a DNSKEY with the SEP bit set to sign all RRsets in the zone (other than the DNSKEY RRset). It is also possible to use a single DNSKEY, with or without the SEP bit set, to sign the entire zone, including the DNSKEY RRset itself.

\subsection{Errors in Examples}

The text in Appendix C.1 of [RFC4035] refers to the examples in Appendix B.1 as "x.w.example.com" while B.1 uses "x.w.example". This is painfully obvious in the second paragraph where it states that the RRSIG labels field value of 3 indicates that the answer was not the result of wildcard expansion. This is true for "x.w.example" but not for "x.w.example.com", which of course has a label count of 4 (antithetically, a label count of 3 would imply the answer was the result of a wildcard expansion).

The first paragraph of Appendix C.6 of [RFC4035] also has a minor error: the reference to "a.z.w.w.example" should instead be "a.z.w.example", as in the previous line.

\subsection{Errors in RFC 5155}

An NSEC3 record that matches an Empty Non-Terminal effectively has no type associated with it. This NSEC3 record has an empty type bit map. Section 3.2.1 of [RFC5155] contains the statement:

Blocks with no types present MUST NOT be included.

However, the same section contains a regular expression:

Type Bit Maps Field $=($ Window Block \# | Bitmap Length | Bitmap )+

The plus sign in the regular expression indicates that there is one or more of the preceding element. This means that there must be at least one window block. If this window block has no types, it contradicts with the first statement. Therefore, the correct text in Section 3.2.1 of [RFC5155] should be:

Type Bit Maps Field = ( Window Block \# | Bitmap Length | Bitmap) *

7. Security Considerations

This document adds SHA-2 and NSEC3 support to the core DNSSEC protocol. Security considerations for those features are discussed in the documents defining them. Additionally, this document addresses some ambiguities and omissions in the core DNSSEC documents that, if not recognized and addressed in implementations, could lead 
to security failures. In particular, the validation algorithm clarifications in section 4 are critical for preserving the security properties DNSSEC offers. Furthermore, failure to address some of the interoperability concerns in section 5 could limit the ability to later change or expand DNSSEC, including adding new algorithms.

The recommendation in Section 5.9 to always set the CD bit has security implications. By setting the CD bit, a resolver will not benefit from more stringent validation rules or a more complete set of trust anchors at an upstream validator.

8. References

8.1. Normative References

[RFC1034] Mockapetris, P., "Domain names - concepts and facilities", STD 13, RFC 1034, November 1987.

[RFC2119] Bradner, S., "Key words for use in RFCs to Indicate Requirement Levels", BCP 14, RFC 2119, March 1997.

[RFC3225] Conrad, D., "Indicating Resolver Support of DNSSEC", RFC 3225, December 2001 .

[RFC4033] Arends, R., Austein, R., Larson, M., Massey, D., and S. Rose, "DNS Security Introduction and Requirements", RFC 4033, March 2005.

[RFC4034] Arends, R., Austein, R., Larson, M., Massey, D., and S. Rose, "Resource Records for the DNS Security Extensions", RFC 4034, March 2005.

[RFC4035] Arends, R., Austein, R., Larson, M., Massey, D., and S. Rose, "Protocol Modifications for the DNS Security Extensions", RFC 4035, March 2005.

[RFC4509] Hardaker, W., "Use of SHA-256 in DNSSEC Delegation Signer (DS) Resource Records (RRs)", RFC 4509, May 2006.

[RFC5155] Laurie, B., Sisson, G., Arends, R., and D. Blacka, "DNS Security (DNSSEC) Hashed Authenticated Denial of Existence", RFC 5155, March 2008.

[RFC5702] Jansen, J., "Use of SHA-2 Algorithms with RSA in DNSKEY and RRSIG Resource Records for DNSSEC", RFC 5702, October 2009 . 


\subsection{Informative References}

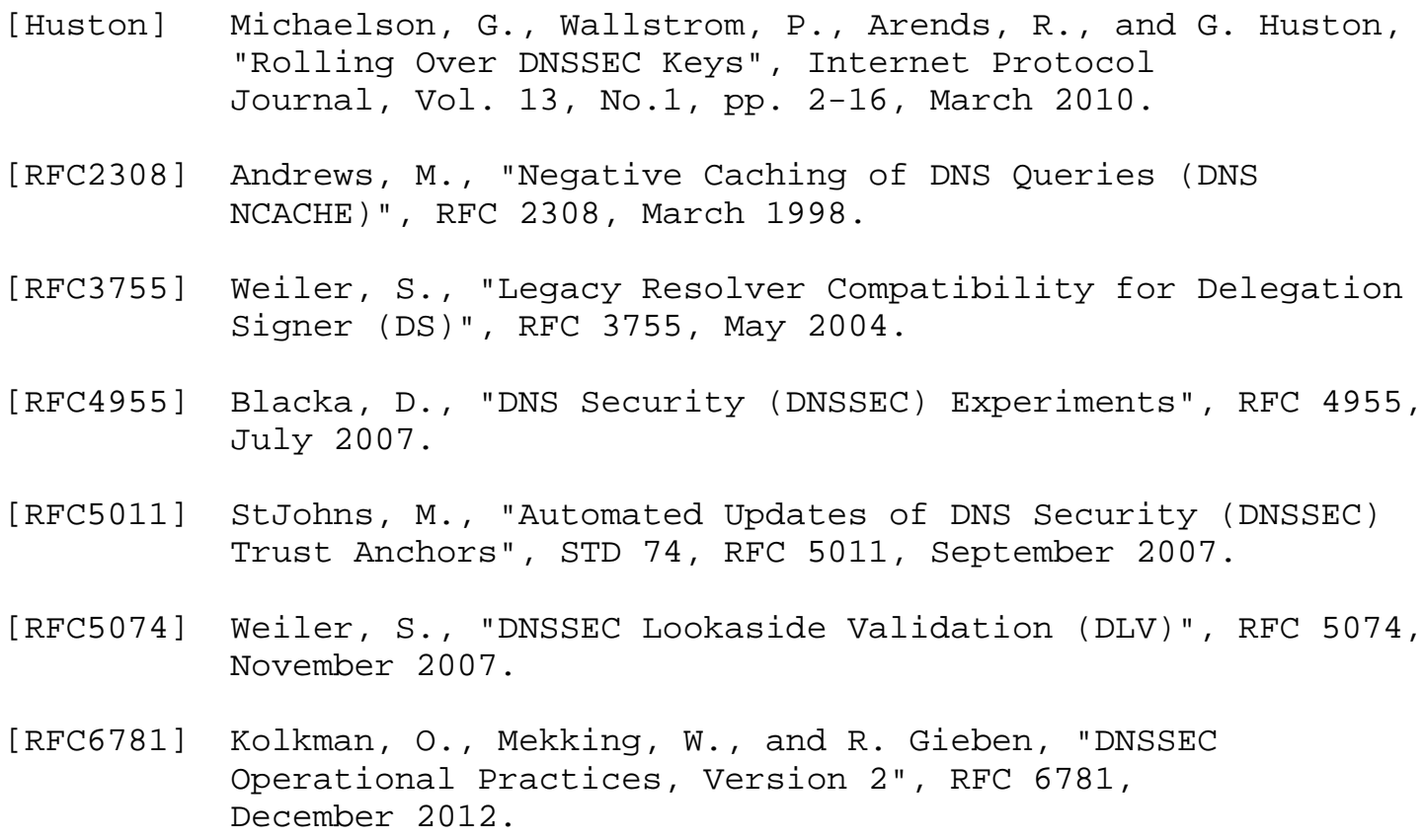


Appendix A. Acknowledgments

The editors would like the thank Rob Austein for his previous work as an editor of this document.

The editors are extremely grateful to those who, in addition to finding errors and omissions in the DNSSEC document set, have provided text suitable for inclusion in this document.

The lack of specificity about handling private algorithms, as described in Section 5.3, and the lack of specificity in handling ANY queries, as described in Section 4.2, were discovered by David Blacka.

The error in algorithm 1 key tag calculation, as described in Section 5.5, was found by Abhijit Hayatnagarkar. Donald Eastlake contributed text for Section 5.5.

The bug relating to delegation NSEC RR's in Section 4.1 was found by Roy Badami. Roy Arends found the related problem with DNAME.

The errors in the [RFC4035] examples were found by Roy Arends, who also contributed text for section 6.3 of this document.

Text on the mandatory algorithm rules was derived from suggestions by Matthijs Mekking and Ed Lewis.

The CD bit logic was analyzed in depth by David Blacka, Olafur Gudmundsson, Mike St. Johns, and Andrew Sullivan.

The editors would like to thank Alfred Hoenes, Ed Lewis, Danny Mayer, Olafur Gudmundsson, Suzanne Woolf, Rickard Bellgrim, Mike St. Johns, Mark Andrews, Wouter Wijngaards, Matthijs Mekking, Andrew Sullivan, Jeremy Reed, Paul Hoffman, Mohan Parthasarathy, Florian Weimer, Warren Kumari and Scott Rose for their contributions to this document.

Appendix B. Discussion of Setting the CD Bit

[RFC4035] may be read as relying on the implicit assumption that there is at most one validating system between the stub resolver and the authoritative server for a given zone. It is entirely possible, however, for more than one validator to exist between a stub resolver and an authoritative server. If these different validators have disjoint trust anchors configured, then it is possible that each would be able to validate some portion of the DNS tree, but neither 
is able to validate all of it. Accordingly, it might be argued that it is desirable not to set the $\mathrm{CD}$ bit on upstream queries, because that allows for maximal validation.

In Section 5.9 of this document, it is recommended to set the CD bit on an upstream query even when the incoming query arrives with $\mathrm{CD}=0$. This is for two reasons: it encourages a more predictable validation experience as only one validator is always doing the validation, and it ensures that all DNSSEC data that exists may be available from the local cache should a query with $\mathrm{CD}=1$ arrive.

As a matter of policy, it is possible to set the CD bit differently than suggested in Section 5.9. A different choice will, of course, not always yield the benefits listed above. It is beyond the scope of this document to outline all of the considerations and counter considerations for all possible policies. Nevertheless, it is possible to describe three approaches and their underlying philosophy of operation. These are laid out in the tables below.

The table that describes each model has five columns. The first column indicates the value of the $\mathrm{CD}$ bit that the resolver receives (for instance, on the name server side in an iterative resolver, or as local policy or from the API in the case of a stub). The second column indicates whether the query needs to be forwarded for resolution (F) or can be satisfied from a local cache (C). The third column is a line number, so that it can be referred to later in the table. The fourth column indicates any relevant conditions at the resolver, for example, whether the resolver has a covering trust anchor, and so on. If there are no parameters here, the column is empty. The fifth and final column indicates what action the resolver takes.

The tables differentiate between "cached data" and "cached RCODE=2". This is a shorthand; the point is that one has to treat $\mathrm{RCODE}=2$ (server failure) as special, because it might indicate a validation failure somewhere upstream. The distinction is really between "cached RCODE=2" and "cached everything else".

The tables are probably easiest to think of in terms of describing what happens when a stub resolver sends a query to an intermediate resolver, but they are perfectly general and can be applied to any validating resolver.

Model 1: "always set"

This model is so named because the validating resolver sets the CD bit on queries it makes regardless of whether it has a covering trust anchor for the query. The general philosophy represented by this 
table is that only one resolver should be responsible for validation irrespective of the possibility that an upstream resolver may be present with trust anchors that cover different or additional QNAMEs. It is the model recommended in section 5.9 of this document.

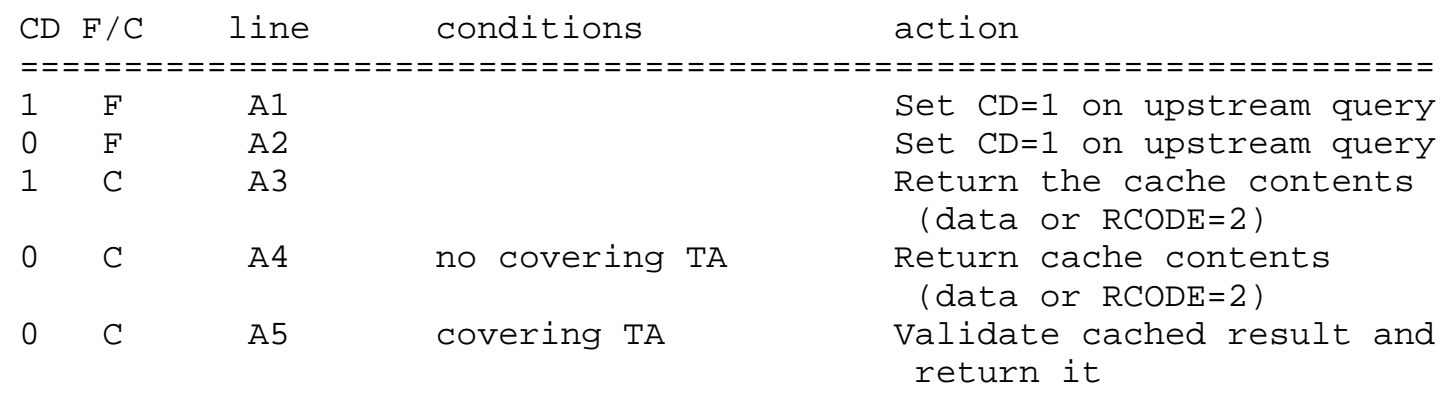

Model 2: "never set when receiving $\mathrm{CD}=0$ "

This model is so named because it sets $\mathrm{CD}=0$ on upstream queries for all received $\mathrm{CD}=0$ queries, even if it has a covering trust anchor. The general philosophy represented by this table is that more than one resolver may take responsibility for validating a QNAME and that a validation failure for a QNAME by any resolver in the chain is a validation failure for the query. Using this model is NOT RECOMMENDED.

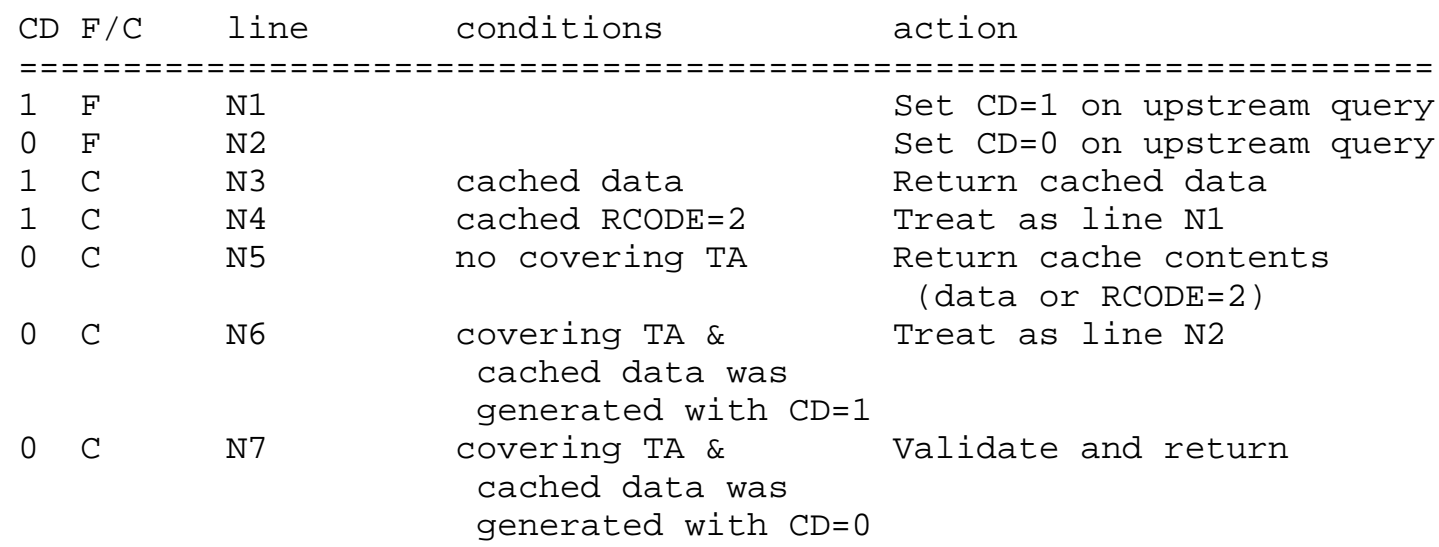

Model 3: "sometimes set"

This model is so named because it sets the CD bit on upstream queries triggered by received $\mathrm{CD}=0$ queries, based on whether the validator has a trust anchor configured that covers the query. If there is no covering trust anchor, the resolver clears the CD bit in the upstream 
query. If there is a covering trust anchor, the resolver sets $C D=1$ and performs validation itself. The general philosophy represented by this table is that a resolver should try and validate QNAMEs for which it has trust anchors and should not preclude validation by other resolvers for QNAMEs for which it does not have covering trust anchors. Using this model is NOT RECOMMENDED.

\begin{tabular}{|c|c|c|c|c|}
\hline $\mathrm{CD}$ & $\mathrm{F} / \mathrm{C}$ & line & conditions & action \\
\hline & 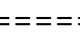 & $====$ & $==============$ & 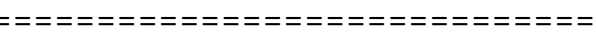 \\
\hline 1 & $\mathrm{~F}$ & S1 & & Set $C D=1$ on upstream query \\
\hline 0 & $\mathrm{~F}$ & S2 & covering TA & Set $C D=1$ on upstream query \\
\hline 0 & $\mathrm{~F}$ & S3 & no covering TA & Set $C D=0$ on upstream query \\
\hline 1 & $\mathrm{C}$ & S4 & cached data & Return cached data \\
\hline 1 & $\mathrm{C}$ & S5 & cached $\mathrm{RCODE}=2$ & Treat as line S1 \\
\hline 0 & $\mathrm{C}$ & S 6 & $\begin{array}{l}\text { cached data was } \\
\text { generated with } \\
C D=0\end{array}$ & Return cache contents \\
\hline 0 & $\mathrm{C}$ & S7 & $\begin{array}{l}\text { cached data was } \\
\text { generated with } \\
\text { CD }=1 \& \\
\text { covering TA }\end{array}$ & $\begin{array}{l}\text { Validate \& return cache } \\
\text { contents }\end{array}$ \\
\hline 0 & $\mathrm{C}$ & S8 & cached $\mathrm{RCODE}=2$ & Return cache contents \\
\hline 0 & $\mathrm{C}$ & S 9 & $\begin{array}{l}\text { cached data } \\
\text { was generated } \\
\text { with } C D=1 \& \\
\text { no covering } \\
\text { TA }\end{array}$ & Treat as line S3 \\
\hline
\end{tabular}

Appendix C. Discussion of Trust Anchor Preference Options

This section presents several different policies for validating resolvers to use when they have a choice of trust anchors available for validating a given answer.

\section{C.1. Closest Encloser}

One policy is to choose the trust anchor closest to the QNAME of the response. For example, consider a validator configured with trust anchors for "example." and "zone.example." When asked to validate a response for "www.sub.zone.example.", a validator using the "Closest Encloser" policy would choose the "zone.example." trust anchor.

This policy has the advantage of allowing the operator to trivially override a parent zone's trust anchor with one that the operator can validate in a stronger way, perhaps because the resolver operator is 
affiliated with the zone in question. This policy also minimizes the number of public key operations needed, which is of benefit in resource-constrained environments.

This policy has the disadvantage of giving the user some unexpected and unnecessary validation failures when sub-zone trust anchors are neglected. As a concrete example, consider a validator that configured a trust anchor for "zone.example." in 2009 and one for "example." in 2011. In 2012, "zone.example." rolls its Key Signing Key (KSK) and updates its DS records, but the validator operator doesn't update its trust anchor. With the "Closest Encloser" policy, the validator gets validation failures.

\section{C.2. Accept Any Success}

Another policy is to try all applicable trust anchors until one gives a validation result of Secure, in which case the final validation result is secure. If and only if all applicable trust anchors give a result of Insecure, the final validation result is Insecure. If one or more trust anchors lead to a Bogus result and there is no secure result, then the final validation result is Bogus.

This has the advantage of causing the fewest validation failures, which may deliver a better user experience. If one trust anchor is out of date (as in our above example), the user may still be able to get a Secure validation result (and see DNS responses).

This policy has the disadvantage of making the validator subject to the compromise of the weakest of these trust anchors, while making it relatively painless to keep old trust anchors configured in perpetuity.

\section{C.3. Preference Based on Source}

When the trust anchors have come from different sources (e.g., automated updates ([RFC5011]), one or more DNSSEC Lookaside Validation (DLV) registries ([RFC5074]), and manual configuration), a validator may wish to choose between them based on the perceived reliability of those sources. The order of precedence might be exposed as a configuration option.

For example, a validator might choose to prefer trust anchors found in a DLV registry over those manually configured on the theory that the manually configured ones will not be as aggressively maintained. 
Conversely, a validator might choose to prefer manually configured trust anchors over those obtained from a DLV registry on the theory that the manually configured ones have been more carefully authenticated.

Or the validator might do something more complex: prefer a sub-set of manually configured trust anchors (based on a configuration option), then trust anchors that have been updated using the mechanism in [RFC5011], then trust anchors from one DLV registry, then trust anchors from a different DLV registry, then the rest of the manually configured trust anchors.

Authors' Addresses

Samuel Weiler (editor)

SPARTA, Inc.

7110 Samuel Morse Drive

Columbia, MD 21046

US

EMail: weiler@tislabs.com

David Blacka (editor)

Verisign, Inc.

12061 Bluemont Way

Reston, VA 20190

US

EMail: davidb@verisign.com 